Dr. med.

Brigitte Moreano Stellvertretende Chefredakteurin brigitte.moreano@ springer.com

\section{Kohortenstudie}

\section{Aktive Kinder werden seltener depressiv}

Bewegen sich Kinder viel, entwickeln sie seltener Depressionen. In der „Trondheim Early Secure Study“" wurden 800 Kinder im Alter von sechs Jahren alle zwei Jahre bis zum zehnten Lebensjahr auf Depressionssymptome hin untersucht. Zudem wurde jeweils eine Woche lang mittels Aktimeter ihre körperliche Bewegung erfasste. Depressive Symptome mit acht und zehn Jahren korrelierten mit einer geringeren körperlichen Aktivität sowohl zum Untersuchungszeitpunkt als auch zwei Jahre zuvor. Bewegungsmangel scheint also einer kind-

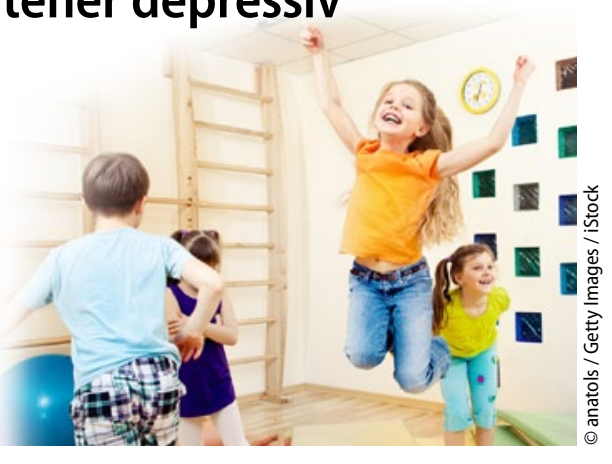

lichen Depression vorauszugehen und nicht umgekehrt.

- Pediatrics 2017; online 9. Januar 2017; e20161711

\title{
Deutsche Registerdaten
}

\section{Depression so schädlich wie traditionelle Herz-Kreislauf-Risiken}

Depressive Verstimmungen sind ein eigenständiger Risikofaktor für eine erhöhte Mortalität, wie eine Auswertung der Augsburger MONICA- und KORA-Register mit 3.428 Männer zeigt. In der Basisuntersuchung war 1.164 Patienten eine depressive Verstimmung attestiert worden. 557 Teilnehmer starben im Laufe von zehn Jahren, davon 269 an kardiovaskulären Ursachen.
Das relative Risiko für einen kardiovaskulär bedingten Tod war bei Depressiven um 53\% erhöht - ähnlich stark wie bei Hypercholesterinämie (+36\%) und Adipositas (+43\%). Der Risikozuwachs bei der Mortalität insgesamt war sogar größer als mit den etablierten Risikofaktoren ( $+53 \%$ vs. $+3 \%$ und $+26 \%$ ).

- Atherosclerosis, in press, online Dezember 2016

\section{Normoton?}

\section{Bei jedem Achten trügt die Praxismessung!}

Ein normaler Blutdruck in der Praxis kann in falscher Sicherheit wiegen. Laut einer US-amerikanischen Erhebung leidet jeder achte Patient mit normotonen Werten in der Praxis an einer maskierten Hypertonie (MHT). Ausgewertet wurden die Daten der Masked Hypertension-Studie (MHTS), der National Health and Nutrition Examination Survey (NHANES) sowie der Improving the Detection of Hypertension (IDH)-Studie.

Die Studienautoren errechneten, dass in den USA mehr als 17 Millionen Erwachsene, also 12,3\% der Bevölkerung an einem nicht erkannten, weil mit der Praxismessung nicht $\mathrm{zu}$ diagnostizierenden Bluthochdruck leiden. Die MHT-Prävalenz war bei Über-45-Jährigen, Männern, Diabetikern und bei Menschen mit prähypertensiven Blutdruckwerten in der Praxis am höchsten. Jetzt müsse in Studien überprüft werden, so die Forderung der US-amerikanischen Kardiologen, bei welchen Personen eine Langzeitblutdruckmessung trotz normaler Blutdruckwerte angezeigt ist.

- American Journal of Epidemiology, online Januar 2017 zusammenlebten. Im Lauf von 18 Jahren ließen sich $16 \%$ von ihnen scheiden, $0,3 \%$ verwitweten. Männer entwickelten nach der Scheidung sechsfach häufiger Alkoholprobleme als solche, die verheiratet blieben. Bei Frauen war die Rate 7,3-fach erhöht. War ein Todesfall Grund für den Partnerverlust, lag die Rate an Alkoholstö- rungen bei dem 3,9-Fachen (Männer) und 4,1-Fachen (Frauen). Ein Viertel der Geschiedenen fand im Studienverlauf einen neuen Ehepartner. Bei ihnen war die Rate an Alkoholproblemen anschließend nur noch halb so hoch wie bei den weiter in Scheidung Lebenden.

- AJP 2017, epub 20.1.17, DOI: 10.1176/appi. ajp.2016.16050589 